Pacific

Journal of

Mathematics

ON ANTIPODES ON A MANIFOLD ENDOWED WITH A GENERIC RIEMANNIAN METRIC

JoËL ROUYER

Volume $212 \quad$ No. 1

November 2003 


\title{
ON ANTIPODES ON A MANIFOLD ENDOWED WITH A GENERIC RIEMANNIAN METRIC
}

\author{
JoËL ROUYER
}

\begin{abstract}
We prove that a generic point of a $C^{r}$ manifold endowed with a generic Riemannian structure has an unique antipode (i.e., farthest point). Furthermore, in the case of 2-dimensional manifolds, such a point is joined to its antipode by at most three minimizing geodesics $(r \geq 2)$.
\end{abstract}

\section{Introduction.}

In a compact metric space $(X, d)$, we call an antipode of a point $p \in X$, any point which realizes the maximum of the distance from $x$. Some conjectures about antipodes were formulated by H. Steinhauss [2]. As an example: Is the sphere the only surface on which each point admits a single antipode and such that the antipodal function is an involution? This question was recently solved by C. Vîlcu who exhibits some counterexamples [6]. Now, it is quite natural to investigate the generic case.

In the case of a convex surface $S$, Tudor Zamfirescu proves in [7] that a generic point of $S$ has an unique antipode, and is joined to it by exactly three segments (A segment, or a minimizing geodesic, is simply a path whose length equals the distance between its extremities).

The aim of this paper is to give an analogous result in the frame of Riemannian geometry. We obtain that the generic uniqueness holds for a $C^{r}$ manifold (of any dimension) endowed with a generic Riemannian metric (Theorem 1), and the fact that at most three geodesics go from a generic point to its antipode holds for such 2-dimensional manifolds (Theorem 2) $(r \geq 2)$.

The first result is optimal, in sense that it fails if you remove any occurrence of "generic". The projective plane with constant curvature is obviously a counterexample to the attempt at deletion of the second occurrence. Concerning the first one, we only need to notice that any sufficiency "long" space admits points with more than one antipode. The antipodes, in such a space, are localized near its "extremities". Any point near enough one extremity has all its antipodes near the other one. By semicontinuity of the antipodal function (see Lemma 5), there exists a point which should have (at least) one antipode near each extremity. 
However, in the case of convex surfaces [8, Theorem 4], as in the case of manifolds homeomorphic to the 2-dimensional sphere [5], a stronger result exists.

\section{Baire categories notion of genericity.}

For elementary results about Baire categories, we refer to any topology book. We simply recall that a subset of topological space is said to be of first category or meager if it is included in a countable union of closed sets with empty interior. A Baire space is a topological space where meager subsets have empty interior. Baire's theorem states that complete metric spaces are Baire spaces. In a Baire space, we say that a generic point satisfies a property, if all points in a residual subset (i.e., a subset whose complement is meager) satisfy this property.

Now we need to endow the set $G^{r}$ of all $C^{r}$ Riemannian structures on a given manifold $M$, with a topology which makes it a Baire space. This can be done in several slightly different ways (see [1] for an another construction).

We fix $g_{0} \in G^{r}$. On one hand, $g_{0}$ provides a norm \|\|$_{x}$, on each fibre over $x$ of the vector bundle

$$
B_{n}=\overbrace{T M^{*} \otimes \cdots \otimes T M^{*}}^{n \text { times }} \otimes T M^{*} \odot T M^{*},
$$

where $\odot$ denotes the symmetric tensor product. As $M$ is compact, we can define a norm \|\|$_{n}$ on the set $\Gamma^{r}\left(B_{n}\right)$ of $C^{r}$ sections of $B_{n}$ by

$$
\|\cdot\|_{n}=\sup _{x \in M}\|\cdot\|_{x} .
$$

On the other hand, $g_{0}$ supplies its Levi-Cività covariant derivation $\nabla$, from $\Gamma^{r}\left(B_{n}\right)$ to $\Gamma^{r-1}\left(B_{n+1}\right)$. Put $\nabla^{p}=\nabla \circ \cdots \circ \nabla$, and define for $g \in G^{\prime r} \stackrel{\text { def }}{=}$ $\Gamma^{r}\left(B_{0}\right)$

$$
\|g\|_{C^{r}}=\max _{p=0, \ldots, r}\left\|\nabla^{p} g\right\|_{p} .
$$

It is obvious that this real valued map is a norm on $G^{\prime r}$. Moreover $G^{\prime r}$ endowed with this norm is a Banach space. For $C^{\infty}$ manifolds, we define the metric

$$
d^{\infty}\left(g, g^{\prime}\right)=\sum_{r=0}^{\infty} 2^{-r} \min \left(1,\left\|g-g^{\prime}\right\|_{C^{r}}\right) .
$$

It is well-known that $\left(G^{\prime \infty}, d^{\infty}\right)$ is a complete metric space whose topology equals the one defined by the norm family $\left(\|\|_{C^{r}}\right)_{r \geq 0}$. Moreover, the topology induced by \|\|$_{C^{r}}$ does not depend on $g_{0} \in G^{r}$.

The set $G^{r}$ of $C^{r}$ Riemannian structures can be defined by

$$
G^{r}=\left\{g \in G^{\prime r} \mid \forall x \in T M, g(x, x)>0 \Leftrightarrow x \neq 0\right\} .
$$


It is obvious that $G^{r}$ is open in $G^{\prime r}$, and so, is a Baire space.

\section{Some continuity or upper semicontinuity results.}

3.1. Some notation. As a matter of geometric notation, we define for $g \in G^{r}, L^{g}(\gamma)$ as the $g$-length of a curve $\gamma, \mathfrak{A}_{x}^{g}$ as the set of $g$-antipodes of a point $x \in M, \mathfrak{D}_{x}^{g}$ as the distance between $x$ and its $g$-antipodes. The $g$-unit tangent bundle over $M$ will be denoted by $T^{1 g} M$. We also denote by $D$ the set of all metrics on $M$ which induce its topology. $D$ is endowed by the metric $\delta$ defined by

$$
\delta\left(d, d^{\prime}\right)=\max _{(x, y) \in M^{2}}\left|d(x, y)-d^{\prime}(x, y)\right| .
$$

For $g \in G^{r}$, we denote by $d^{g} \in D$ the metric corresponding to the Riemannian structure $g$. At last, we define $\Sigma_{x y}^{g}$ as the set of $g$-segments from $x$ to $y$, and $A_{2}^{g}$ as the set of points of $M$ which admit at least two $g$-antipodes.

We also need more abstract notations. For any metric space $(X, d)$ we define $\mathcal{H}(X)$ as the set of all nonempty compact subsets of $X$, which is endowed with the well-known Hausdorff metric, denoted by the same symbol $d$ :

$$
\begin{aligned}
d\left(K_{1}, K_{2}\right) & =\max \left(d^{\complement}\left(K_{1}, K_{2}\right), d^{\subset}\left(K_{2}, K_{1}\right)\right), \\
d^{\complement}\left(K_{1}, K_{2}\right) & =\max _{x \in K_{1}} \min _{y \in K_{2}} d(x, y) .
\end{aligned}
$$

It is well-known that $\mathcal{H}(X)$ is compact whenever $X$ is compact. An $\mathcal{H}(X)$ valued function is said to be upper semicontinuous, if it is continuous for the topology induced by $d^{\complement}$. Note that $d^{\complement}$ satisfies the triangle inequality, and $d^{\complement}\left(K_{1}, K_{2}\right)$ vanishes if and only if $K_{1} \subset K_{2}$.

Given a subset $P$ of $X$, and a positive real number $\rho$, we denote by $P+\rho$ the union of all open balls of radius $\rho$ centered at the elements of $P$.

We define $\mathcal{H} \mathcal{G}$ as the set of compact metric spaces up to isometries. Given two spaces $X, Y \in \mathcal{H G}$, we say that $X$ is included in $Y$, and write $X \subset Y$, if there exists an isometric injective map from $X$ into $Y$. This defines a partial order on $\mathcal{H G}$. Now we put for $\left(X, d_{X}\right),\left(Y, d_{Y}\right) \in \mathcal{H} \mathcal{G}$

$$
\begin{aligned}
& d_{H G}^{\complement}(X, Y)=\inf _{\left(Z, d_{z}\right), \phi, \psi} d_{Z}^{\complement}(\phi(X), \psi(Y)) \\
& d_{H G}(X, Y)=\max \left(d_{H G}^{\subset}(X, Y), d_{H G}^{\subset}(Y, X)\right),
\end{aligned}
$$

where the infimum is taken over all metric spaces $\left(Z, d_{Z}\right)$, and all isometric injective maps, $\phi$, from $X$ to $Z$, and, $\psi$, from $Y$ to $Z$. $d_{H G}$ is nothing but the well-known Hausdorff-Gromov metric on $\mathcal{H} \mathcal{G}[\mathbf{3}]$. A $\mathcal{H} \mathcal{G}$-valued function is said to be upper semicontinuous if it is continuous for the topology induced by $d_{H G}^{\complement}$. It is easy to see that $d_{H G}^{\subset}$ satisfies the triangle inequality, moreover we have: 
Lemma 1. Let $X, Y \in \mathcal{H G}$. We have $d_{H G}^{\subset}(X, Y)=0$ if and only if $X \subset Y$. Proof. It is clear that $X \subset Y$ implies $d_{H G}^{\subset}(X, Y)=0$. Conversely, if $d_{H G}^{\subset}(X, Y)=0$, then there exist metric spaces $\left(Z_{n}, d_{n}\right)$, and injective isometries $\phi_{n}: X \rightarrow Z_{n}$ and $\psi_{n}: Y \rightarrow Z_{n}$ such that $d^{\complement}\left(\phi_{n}(X), \psi_{n}(Y)\right)<\frac{1}{n}$. Let $Z$ be a coproduct of all compact metric spaces $Z_{n}^{\prime} \stackrel{\text { def }}{=} \phi_{n}(X) \cup \psi_{n}(Y)$, $n \in \mathbb{N}$. We define on $Z$ an equivalence relation $R$ by $z R z^{\prime}$ if and only if both $z$ and $z^{\prime}$ are the image of the same point $y \in Y$ by $\psi_{n}$ for some $n \in \mathbb{N}$. Put $\psi=s \circ i_{n} \circ \psi_{n}$, with $i_{n}: Z_{n}^{\prime} \rightarrow Z$ the canonical injection, and $s: Z \rightarrow Z / R$ the canonical surjection. $Z / R$ is a metric space with the metric $d_{Z}$ defined by

$$
\begin{gathered}
d_{Z / R}\left(z_{n}, z_{m}\right)=\inf _{y \in Y}\left(d_{n}\left(z_{n}, \psi(y)\right)+d_{m}\left(z_{m}, \psi(y)\right)\right), \\
\text { for } z_{i} \in Z_{i}, i=n \text { or } m, n \neq m \\
d_{Z / R}\left(z_{n}, z_{n}^{\prime}\right)=d_{n}\left(z_{n}, z_{n}^{\prime}\right), \text { for } z_{n}, z_{n}^{\prime} \in Z_{n} .
\end{gathered}
$$

We claim that $Z / R$ is compact. Let $\left(z_{p}\right)=\left(s\left(z_{p}^{\prime}\right)\right)$ be a sequence of $Z / R$. $z_{p}^{\prime}$ belongs to $i_{n_{p}}\left(Z_{n_{p}}^{\prime}\right)$ for some integer $n_{p}$, and then, there exists a point $y_{p} \in \psi(Y)$ such that $d_{Z / R}\left(z_{p}, y_{p}\right) \leq \frac{1}{n_{p}}$. If the sequence $\left(n_{p}\right)$ is bounded by an integer $N$, then we can select from $\left(z_{p}\right)$ a converging subsequence, by compactness of $Z_{1}^{\prime}, Z_{2}^{\prime}, \ldots, Z_{N}^{\prime}$, else, we can assume by selecting suitable subsequences, that $\lim _{p} \frac{1}{n_{p}}=0$. As $Y$ is compact, we can select from $\left(y_{p}\right)$, and so from $\left(z_{p}\right)$ too, a converging subsequence. This proves the claim. Now, as $s \circ i_{n} \circ \phi_{n}$ are isometries (hence, form an equicontinuous family), we can extract a subsequence converging to an isometry $\phi: X \rightarrow \psi(Y)$. This completes the proof.

Given a compact metric space $X$, we denote by $j_{X}$ the canonical map from $\mathcal{H}(X)$ to $\mathcal{H G}$. Of course, $\mathfrak{j}_{X}$ is upper semicontinuous and order preserving.

Let $X \in \mathcal{H G}$ and $P \subset \mathcal{H G}$, we will denote by $d_{H G}^{\subset}(X, P)$ the infimum $\inf _{Y \in P} d_{H G}^{\subset}(X, Y)$. As $d_{H G}^{\subset}$ is not a metric, the following lemma is not obvious.

Lemma 2. Let $P$ be a compact subset of $\mathcal{H} \mathcal{G}$, take $X \in \mathcal{H G}$. Then $d_{H G}^{\subset}(X$, $P)=0$ if and only if there exists a compact metric space $Y \in P$ such that $X \subset Y$.

Proof. Given an integer $n$, there exists a metric space $Y_{n} \in P$ such that $d_{H G}^{\subset}\left(X, Y_{n}\right)<\frac{1}{n}$. Let $Y$ be the limit of a converging subsequence of $\left(Y_{n}\right)$. We have $d_{H G}^{\subset}(X, Y) \leq d_{H G}^{\subset}\left(X, Y_{n}\right)+d_{H G}\left(Y_{n}, Y\right)$. As the right-hand side tends to zero, left-hand side must vanish.

We have to consider a special subset $\mathcal{T} \subset \mathcal{H G}$, which is the set of those metric spaces, included in the unit circle (i.e., $\mathbb{R} / 2 \pi \mathbb{Z}$ ), whose cardinality is at most 3 . It is clear that $\mathcal{T}$ is compact. 
3.2. Useful lemmas. This miscellany is nothing but the list of lemmas we need.

Lemma 3. The map from $G^{r}$ to $D$ which associates to $g$ the corresponding metric $d^{g}$ is locally Lipschitz continuous with respect to the metric $\delta$ and the norm \|\|$_{C^{0}}$.

Proof. We denote by $N(g)$ the real number $\sup _{x \in T^{1 g_{0} M}}\left|\frac{1}{g(x, x)}\right|$. Consider two Riemannian structures $g$ and $g^{\prime}=g+h$. Let $x, y$ be two points of $M$, such that $\delta\left(d^{g}, d^{g^{\prime}}\right)=d^{g^{\prime}}(x, y)-d^{g}(x, y)$ (you may exchange $g$ and $g^{\prime}$ if necessary). Let $\sigma$ be a $g$-segment from $x$ to $y$. We have

$$
\begin{aligned}
d^{g^{\prime}}(x, y) & \leq L^{g^{\prime}}(\sigma) \\
& =\int \sqrt{g+h}(\dot{\sigma}(t), \dot{\sigma}(t)) d t \\
& \leq \int \sqrt{g}(\dot{\sigma}(t), \dot{\sigma}(t)) d t+\frac{1}{2} \int \frac{|h|}{\sqrt{g}}(\dot{\sigma}(t), \dot{\sigma}(t)) d t \\
& \leq L^{g}(\sigma)+\frac{1}{2}\|h\|_{C^{0}} N(g) \int \sqrt{g}(\dot{\sigma}(t), \dot{\sigma}(t)) d t \\
& \leq d^{g}(x, y)+\frac{1}{2}\|h\|_{C^{0}} N(g) L^{g}(\sigma) \\
& \leq d^{g}(x, y)+\frac{1}{2}\|h\|_{C^{0}} N(g) \operatorname{diam}(M, g) .
\end{aligned}
$$

Hence

$$
\begin{aligned}
\delta\left(d^{g}, d^{g^{\prime}}\right) & =d^{g^{\prime}}(x, y)-d^{g}(x, y) \\
& \leq \frac{1}{2}\|h\|_{C^{0}} N(g) \operatorname{diam}(M, g) .
\end{aligned}
$$

Lemma 4. The map $\mathfrak{D}$ from $G^{r} \times M$ to $\mathbb{R}$ is locally Lipschitz continuous with respect to both variables.

Proof. Let $x, x^{\prime}$ be in $M, g, g^{\prime}$ be in $G^{r}$, and take a $g$-antipode $y$ of $x$.

$$
\begin{aligned}
\mathfrak{D}_{x}^{g} & =d^{g}(x, y) \\
& \leq d^{g^{\prime}}\left(x^{\prime}, y\right)+d^{g}\left(x, x^{\prime}\right)+\delta\left(d^{g}, d^{g^{\prime}}\right) \\
& \leq \mathfrak{D}_{x^{\prime}}^{g^{\prime}}+d^{g}\left(x, x^{\prime}\right)+\delta\left(d^{g}, d^{g^{\prime}}\right) .
\end{aligned}
$$

Of course, the same holds when you exchange $(x, g)$ and $\left(x^{\prime}, g^{\prime}\right)$. Lemma 3 completes the proof.

Lemma 5. The map $\mathfrak{A}$ from $G^{r} \times M$ to $\mathcal{H}(M)$ is upper semicontinuous. 
Proof. Suppose the result fails. There exists a real number $\varepsilon>0$, such that for each integer $n$, there exists a point $x_{n}$ and a Riemannian structure $g_{n}$ such that

$$
\begin{aligned}
\left\|g-g_{n}\right\|_{C^{r}} & <\frac{1}{n} \\
d^{g}\left(x, x_{n}\right) & <\frac{1}{n} \\
\mathfrak{A}_{x_{n}}^{g_{n}} & \nsubseteq A_{x}^{g}+\varepsilon .
\end{aligned}
$$

The formula (1) implies the existence of a sequence $\left(y_{n}\right)$ of $g_{n}$-antipodes of $x_{n}$ such that $y_{n} \notin \mathfrak{A}_{x}^{g}+\varepsilon$. Now, select a converging subsequence from $y_{n}$, and denote by $y$ its limit. On one hand, as $\mathfrak{A}_{x}^{g}+\varepsilon$ is open, $y \notin \mathfrak{A}_{x}^{g}$. On the other hand, by Lemmas 4 and 3 , the identity $\mathfrak{D}_{x_{n}}^{g_{n}}=d^{g_{n}}\left(y_{n}, x_{n}\right)$ leads to $\mathfrak{D}_{x}^{g}=d^{g}(y, x)$, and we obtain a contradiction.

Lemma 6. The map

$$
\begin{aligned}
G^{r} \times M \times M & \rightarrow \mathcal{H}(\mathcal{H}(M)) \\
(g, x, y) & \mapsto \Sigma_{x y}^{g}
\end{aligned}
$$

is upper semicontinuous.

Proof. Suppose the result fails. There exists $\varepsilon>0$, and three sequences $\left(g_{n}\right)$, $\left(x_{n}\right)$ and $\left(y_{n}\right)$, converging respectively to $g \in G^{r}, x \in M$, and $y \in M$, such that a $g_{n}$-segment $\sigma_{n}$ from $x_{n}$ to $y_{n}$ satisfying $\min \left\{d^{g}\left(\sigma_{n}, \sigma^{\prime}\right) \mid \sigma^{\prime} \in \Sigma_{x y}^{g}\right\}>\varepsilon$ exists. We can select from $\sigma_{n}$ a converging subsequence which tends to a $g$-segment $\sigma$ from $x$ to $y$, and a contradiction is found.

Lemma 7. Let $(X, d)$ be a metric space, $K$ be a subset of $\mathcal{H G}$, and $F: X \rightarrow$ $\mathcal{H G}$ be an upper semicontinuous function. The map from $X$ to $\mathbb{R}$,

$$
x \mapsto d_{H G}^{\subset}(F(x), K)
$$

is upper semicontinuous.

Proof. Choose $\varepsilon>0, x \in X$, and put $\delta=d_{H G}^{\subset}(F(x), K)$. Choose $\chi \in K$ such that $d_{H G}^{\complement}(F(x), \chi)<\delta+\frac{\varepsilon}{2}$. There exists a compact metric space $\left(Z_{1}, d\right)$ and two isometric injective maps $g_{1}: F(x) \rightarrow Z_{1}$ and $h_{1}: \chi \rightarrow Z_{1}$ such that

$$
g_{1}(F(x)) \subset h_{1}(\chi)+\left(\frac{\varepsilon}{2}+\delta\right) .
$$

By upper semicontinuity of $F$, there exists a real number $\eta>0$, such that for all points $y$ of the open ball $\{x\}+\eta$ we have

$$
d_{H G}^{\subset}(F(y), F(x))<\frac{\varepsilon}{2} .
$$

Hence, there exists a metric space $\left(Z_{2}, d\right)$ and two isometric injective maps $g_{2}: F(x) \rightarrow Z_{2}$ and $f_{2}: F(y) \rightarrow Z_{2}$ such that

$$
f_{2}(F(y)) \subset g_{2}(F(x))+\frac{\varepsilon}{2} .
$$


Let $Z$ be a coproduct of $Z_{1}$ and $Z_{2}$ where $g_{1}(F(x))$ and $g_{2}(F(x))$ have been identified. We obtain three injective isometric maps $f: F(y) \rightarrow Z$, $g: F(x) \rightarrow Z$ and $h: \chi \rightarrow Z$ such that

$$
\begin{gathered}
g(F(x)) \subset h(\chi)+\left(\frac{\varepsilon}{2}+\delta\right) \\
f(F(y)) \subset g(F(x))+\frac{\varepsilon}{2} .
\end{gathered}
$$

It follows that

$$
f(F(y)) \subset h(\chi)+(\varepsilon+\delta) .
$$

We have proved that for all $y \in\{x\}+\eta$,

$$
\begin{aligned}
d_{H G}^{\subset}(F(y), K) & \leq d_{H G}^{\subset}(F(y), \chi) \\
& \leq d_{H G}^{\subset}(F(x), K)+\varepsilon .
\end{aligned}
$$

Lemma 8. Let $(X, d)$ and $(Y, d)$ be metric spaces. Let $F: X \rightarrow \mathcal{H}(Y)$ be an upper semicontinuous function, and $G: \mathcal{H}(Y) \rightarrow \mathcal{H G}$ be an order preserving (for order $\subset$ ) and upper semicontinuous function. Then $G \circ F$ is upper semicontinuous.

Proof. Choose $\varepsilon>0$ and $x \in X$. The upper semicontinuity of $G$ implies the existence of a real number $\varepsilon^{\prime}>0$ such that for all $K$ in $\mathcal{H}(Y)$,

$$
d(K, F(x)) \leq \varepsilon^{\prime} \Longrightarrow d_{H G}^{\subset}(G(K), G \circ F(x))<\varepsilon .
$$

By upper semicontinuity of $F$, there exists a real number $\eta>0$ such that for all $y \in\{x\}+\eta$

$$
F(y) \subset F(x)+\varepsilon^{\prime} .
$$

Put $K_{0}=F(x) \cup F(y)$. As $G$ preserves order, we have

$$
G \circ F(y) \subset G\left(K_{0}\right) \text {. }
$$

As by $(3) d\left(K_{0}, F(x)\right) \leq \varepsilon^{\prime}$, (2) leads to

$$
d_{H G}^{\subset}\left(G\left(K_{0}\right), G \circ F(x)\right)<\varepsilon .
$$

Hence, there exists a metric space $(Z, d)$ and two injective isometric maps $f: G \circ F(x) \rightarrow Z$ and $g: G\left(K_{0}\right) \rightarrow Z$ such that

$$
g \circ G\left(K_{0}\right) \subset f \circ G \circ F(x)+\varepsilon .
$$

This formula and (4) lead to

$$
g \circ G \circ F(y) \subset f \circ G \circ F(x)+\varepsilon .
$$

Hence $d_{H G}^{\subset}(G \circ F(y), G \circ F(x)) \leq \varepsilon$ for all $y \in\{x\}+\eta$, and the lemma is proved. 


\section{Comeback to geometry.}

4.1. Generic uniqueness of antipodes. Now, we can enunciate and prove the theorems described in the introduction.

Theorem 1. Let $M$ be a $C^{r}$ compact manifold $(r=2, \ldots, \infty)$ endowed with a generic Riemannian structure of $G^{r}$. A generic point of $M$ admits an unique antipode.

For proving this, we need the following:

Lemma 9. Let $n$ be a positive integer. The interior of

$$
\mathcal{U}_{x}(n) \stackrel{\text { def }}{=}\left\{g \in G^{r} \mid \operatorname{diam}\left(\mathfrak{A}_{x}^{g}\right) \geq 1 / n\right\}
$$

is empty.

Proof. Let $g$ be in $\mathcal{U}_{x}(n), \rho$ be an integer less or equal to $r$, and $\varepsilon$ be a positive real number. We shall exhibit a Riemannian structure $g^{\prime} \notin \mathcal{U}_{x}(n)$ such that $\left\|g-g^{\prime}\right\|_{C^{\rho}}<\varepsilon$. We denote by $\lambda$ the $g$-injectivity radius at point $x$. Take $y$, a $g$-antipode of $x$. Let $\Sigma=\Sigma_{x y}^{g}$ be the set of segments from $x$ to $y$, with their arc length parameter. We define $S=\{\sigma(\lambda / 2) \mid \sigma \in \Sigma\}$. Let $\Phi$ be the $g$-exponential mapping at $x$. As $\Phi$ is continuous on $T_{x} M$ there exists a real number $\alpha$ such that for all tangent vectors $u, v$ in the ball $\{0\}+2 \mathfrak{D}_{x}^{g}$ of $T_{x} M$

$$
\|u-v\|<\alpha \Longrightarrow d^{g}(\Phi(u), \Phi(v))<\frac{1}{12 n},
$$

where the norm \|\| is the $g$-norm. As, restricted to $\{0\}+\frac{2 \lambda}{3}$, $\Phi$ has a welldefined and continuous inverse, we can find a positive real number $\eta$, such that

$$
\forall z, z^{\prime} \in\{x\}+\frac{2 \lambda}{3}, d^{g}\left(z, z^{\prime}\right)<\eta \Longrightarrow\left\|\Phi^{-1}\left(z^{\prime}\right)-\Phi^{-1}(z)\right\|<\frac{\lambda \alpha}{2 \mathfrak{D}_{x}^{g}} \stackrel{\text { def }}{=} \beta .
$$

Choose a positive $C^{\rho}$ function $\phi$ such that $V \stackrel{\text { def }}{=}\{x \mid \phi(x)>0\}$ satisfies $S \subset V \subset S+\eta$. Now put $g^{\prime}=g(1+\iota \phi)$, with $\iota$ a positive small real number such that:

(i) $\left\|g-g^{\prime}\right\|_{C^{\rho}}<\varepsilon$

(ii) $\delta\left(d^{g}, d^{g^{\prime}}\right)<\min \left(\alpha, \frac{1}{4 n}\right)$.

Consider a $g^{\prime}$-segment $\sigma^{\prime}$ from $x$ to $y$. We have the following inequalities:

$$
\mathfrak{D}_{x}^{g^{\prime}} \geq d^{g^{\prime}}(x, y)=L^{g^{\prime}}\left(\sigma^{\prime}\right) \geq L^{g}\left(\sigma^{\prime}\right) \geq d^{g}(x, y)=\mathfrak{D}_{x}^{g} .
$$

Moreover, either $\sigma^{\prime}$ passes across $V$, and $L^{g^{\prime}}\left(\sigma^{\prime}\right)>L^{g}\left(\sigma^{\prime}\right)$, or $\sigma^{\prime}$ is not a $g$-segment, and $L^{g}\left(\sigma^{\prime}\right)>d^{g}(x, y)$. In both cases, we have

$$
\mathfrak{D}_{x}^{g^{\prime}}>\mathfrak{D}_{x}^{g}
$$


Let $y^{\prime}$ be in $\mathfrak{A}_{x}^{g^{\prime}}$ and $\sigma$ be a $g$-segment from $x$ to $y^{\prime}$. If $\sigma \cap V=\emptyset$, we would have

$$
\mathfrak{D}_{x}^{g} \geq d^{g}\left(x, y^{\prime}\right)=L^{g}(\sigma)=L^{g^{\prime}}(\sigma) \geq d^{g^{\prime}}\left(x, y^{\prime}\right)=\mathfrak{D}_{x}^{g^{\prime}},
$$

which is in contradiction with (5). Hence $\sigma \cap V \neq \emptyset$, and there exists a $g$-segment $\sigma_{0} \in \Sigma$ and a real number $\tau$ such that $d^{g}\left(\sigma_{0}\left(\frac{\lambda}{2}\right), \sigma(\tau)\right)<\eta$. Let $u_{0}, u \in T_{x} M$ be two unit vectors such that $\Phi(t u)=\sigma(t)$ and $\sigma_{0}(t)=\Phi\left(t u_{0}\right)$. We have $\left|\frac{\lambda}{2}-\tau\right| \leq\left\|\frac{\lambda}{2} u_{0}-\tau u\right\|<\beta$. Hence

$$
\begin{aligned}
\left\|\mathfrak{D}_{x}^{g} u_{0}-2 \frac{\mathfrak{D}_{x}^{g}}{\lambda} \tau u\right\| & <\alpha \\
\left\|\mathfrak{D}_{x}^{g} u-2 \frac{\mathfrak{D}_{x}^{g}}{\lambda} \tau u\right\| & <\alpha,
\end{aligned}
$$

and then

$$
\begin{gathered}
d^{g}\left(\sigma_{0}\left(\mathfrak{D}_{x}^{g}\right), \sigma\left(\frac{2 \mathfrak{D}_{x}^{g}}{\lambda} \tau\right)\right)<\frac{1}{12 n} \\
d^{g}\left(\sigma\left(\mathfrak{D}_{x}^{g}\right), \sigma\left(\frac{2 \mathfrak{D}_{x}^{g}}{\lambda} \tau\right)\right)<\frac{1}{12 n} .
\end{gathered}
$$

On the other hand, by Hypothesis (ii), we have

$$
d^{g}\left(x, y^{\prime}\right) \leq \mathfrak{D}_{x}^{g} \leq \mathfrak{D}_{x}^{g^{\prime}}=d^{g^{\prime}}\left(x, y^{\prime}\right)<d^{g}\left(x, y^{\prime}\right)+\alpha,
$$

hence $\left|d^{g}\left(x, y^{\prime}\right)-\mathfrak{D}_{x}^{g}\right|<\alpha$ and

$$
d^{g}\left(\sigma\left(\mathfrak{D}_{x}^{g}\right), \sigma\left(d^{g}\left(x, y^{\prime}\right)\right)\right)<\frac{1}{12 n} .
$$

As $y=\sigma_{0}\left(\mathfrak{D}_{x}^{g}\right)$ and $y^{\prime}=\sigma\left(d^{g}\left(x, y^{\prime}\right)\right),(6),(7)$ and (8) lead to $d^{g}\left(y, y^{\prime}\right)<\frac{1}{4 n}$, which becomes together with Hypothesis (ii) $d^{g^{\prime}}\left(y, y^{\prime}\right)<\frac{1}{2 n}$. This holds for each $g^{\prime}$-antipode $y^{\prime}$ of $x$, hence $\operatorname{diam}^{g^{\prime}}\left(\mathfrak{A}_{x}^{g^{\prime}}\right)<\frac{1}{n}$, and finally $g^{\prime} \notin \mathcal{U}_{x}(n)$.

Proof of Theorem 1. Put $\mathcal{U}^{g}(n)=\left\{x \in M \mid \operatorname{diam}\left(\mathfrak{A}_{x}^{g}\right) \geq 1 / n\right\}$. Lemma 5 implies that $\mathcal{U}^{g}(n)$ and $\mathcal{U}_{x}(n)$ are closed subsets of $M$ and $G^{r}$ respectively. Let $S$ be a countable dense subset of $M$. We have

$$
\begin{aligned}
\left\{g \in G^{r} \mid A_{2}^{g} \text { is not meager }\right\} & =\{g \in G^{r} \mid \exists n \in \mathbb{N}, \overbrace{\mathcal{U}^{g}(n)}^{\circ} \neq \emptyset\} \\
& =\bigcup_{n \in \mathbb{N}}\{g \in G^{r} \mid \overbrace{\mathcal{U}^{g}(n)}^{\circ} \neq \emptyset\} \\
& \subset \bigcup_{n \in \mathbb{N} x \in S}\left\{g \in G^{r} \mid x \in \mathcal{U}^{g}(n)\right\} \\
& =\bigcup_{n \in \mathbb{N} x \in S} \bigcup \mathcal{U}_{x}(n) .
\end{aligned}
$$


Hence, by Lemma $9,\left\{g \in G^{r} \mid A_{2}^{g}\right.$ is not meager $\}$ is meager.

\subsection{Generic number of segments from a point to its antipode.} Given a $C^{r}$ manifold $M$, we denote by $\mathcal{S} \subset G^{r} \times M$ the set of all ordered pairs $(g, x)$ such that $\mathfrak{A}_{x}^{g}$ contains a single point. Let $a: \mathcal{S} \rightarrow M$ be the continuous function which associates to $(g, x)$ the only $g$-antipode of $x$. We define $\mathcal{S}^{g}=M \backslash A_{2}^{g}=\{x \in M \mid(g, x) \in \mathcal{S}\}$ and $\mathcal{S}_{x}=\left\{g \in G^{r} \mid(g, x) \in \mathcal{S}\right\}$.

In order to prove the result concerning surfaces, we have to use the following:

Lemma 10. Let $(M, g)$ be a $C^{r}$ Riemannian 2-dimensional manifold $(r \geq$ 2). Let $x$ be a point of $M$, and $y$ be a g-antipode of $x$. Denote by $\vec{\Sigma} \subset T_{y}^{1 g} M$ the set of unit vectors tangent to a segment from $x$ to $y$.

Then $\vec{\Sigma}$ cannot be included in any open half-plane of $T_{y} M$. This implies that either $\vec{\Sigma}$ has cardinality at least three, or $\vec{\Sigma}=\{-u, u\}$ for a suitable vector $u \in T_{y}^{1 g} M$.

Proof. Assume that $\vec{\Sigma}$ is included in some open half-plane, and let $\gamma:[0, \varepsilon] \rightarrow$ $M$ be a arclength parameterized arc, starting at $y$, and directed by the bisector $u \in T_{y}^{1 g} M$ of the other half-plane. For each integer $n$, there exists a minimizing geodesic $\sigma_{n}$ from $x$ to $\gamma(1 / n)$. By selecting a subsequence, we can assume that $\sigma_{n}$ is tending to a segment $\sigma$ from $x$ to $y$. Let $v$ be the unit tangent vector at $y$ to $\sigma$. Of course $g(u, v)<0$. By a variant of the first variation formula of arclength $L^{g}\left(\sigma_{n}\right)=L^{g}(\sigma)-\frac{1}{n} g(u, v)+o\left(\frac{1}{n}\right)$, hence $y_{n}$ is farther from $x$ than $y$ for $n$ large enough, and we obtain a contradiction.

Now, we can enunciate the following:

Theorem 2. Let $M$ be $C^{r}$ 2-dimensional manifold $(r=2,3, \ldots, \infty)$, endowed with a generic Riemannian structure. A generic point of $M$ is joined to its only antipode by at most three segments.

We need three lemmas.

Lemma 11. We denote by $\vec{\Sigma}_{x y}^{g}$ the set of the g-unit tangent vectors at $y$, to segments from $x$ to $y$. Assume $r \geq 2$. The map $\xi$ from $\mathcal{S}$ to $\mathcal{H} \mathcal{G}$ defined by $\xi(g, x)=\mathfrak{j}_{T^{1 g} M}\left(\vec{\Sigma}_{x a_{x}^{g}}^{g}\right)$, is upper semicontinuous.

Proof. It is obvious, by continuity of $a$, and Lemma 6 , that $(g, x) \longmapsto \Sigma_{x a_{x}^{g}}^{g}$ is upper semicontinous. Now, with hypothesis $r \geq 2$, the convergence of a sequence of geodesics $\left(\sigma_{n}: I \rightarrow M\right)_{n}$ to $\sigma$ with respect to the Hausdorff metric, implies, for a suitable parametrization, the uniform convergence of the derivatives $\left(\dot{\sigma}_{n}: I \rightarrow T M\right)$ to $\dot{\sigma}$. Hence, the map

$$
\tau: \Sigma_{x a_{x}^{g}}^{g} \mapsto \vec{\Sigma}_{x a_{x}^{g}}^{g}
$$


from a suitable subset of $\mathcal{H}(\mathcal{H}(M))$ to $\mathcal{H}(T M)$, is continuous. As $\tau$ preserves order, we obtain by virtue of Lemma 8 that $\mathfrak{j}_{T M} \circ \tau$ is an (order preserving) upper semicontinuous map. Applying once again the same lemma gives the desired result.

Lemma 12. The map $\mu$ from $\mathcal{S}$ to $\mathbb{R}$, defined by

$$
\mu_{x}^{g}=d_{H G}^{\subset}\left(\dot{j}_{T M}\left(\vec{\Sigma}_{x a_{x}^{g}}^{g}\right), \mathcal{T}\right)
$$

is upper semicontinuous.

Proof. This is a consequence of Lemmas 11 and 7.

Denote by $\mathcal{V}(n)$ the set $\left\{(g, x) \in \mathcal{S} \mid \mu_{x}^{g} \geq \frac{1}{n}\right\}$, and put

$$
\begin{aligned}
& \mathcal{V}^{g}(n)=\{x \in M \mid(g, x) \in \mathcal{V}(n)\} \\
& \mathcal{V}_{x}(n)=\left\{g \in G^{r} \mid(g, x) \in \mathcal{V}(n)\right\} .
\end{aligned}
$$

By Lemma $12, \mathcal{V}_{x}(n)$ and $\mathcal{V}^{g}(n)$ are closed subsets of $\mathcal{S}_{x}$ and $\mathcal{S}^{g}$ respectively, moreover we can prove the following:

Lemma 13. Assume $r \geq 2$. $\mathcal{V}_{x}(n)$ has empty interior in $\mathcal{S}_{x}$.

Proof. Fix $x \in M, n \in \mathbb{N}, g \in \mathcal{V}_{x}(n)$ and put $y=a_{x}^{g}$. If $\sigma$ is a curve going from $x$ to $y$, we denote by $\vec{\sigma} \in T_{y}^{1 g} M$, the $g$-unit tangent vector to $\sigma$. The $g$-distance in $T_{y}^{1 g} M$ (i.e., $\left.\arccos g(\cdot, \cdot)\right)$ is denoted by $(\cdot, \cdot)$.

Let $\sigma_{0}$ be a $g$-segment from $x$ to $y$, and choose a positive function $\phi$ : $M \rightarrow \mathbb{R}$ such that $V \stackrel{\text { def }}{=}\{x \in M \mid \phi(x)>0\}$ satisfies:

(i) Each $g$-segment from $x$ to $y$ passing across $V$ satisfies $\left(\vec{\sigma}, \vec{\sigma}_{0}\right)<\frac{1}{4 n}$.

(ii) There exists a real number $\varepsilon>0$, such that all segments from $x$ to $y$ satisfying $\left(\vec{\sigma}, \vec{\sigma}_{0}\right)<\varepsilon$ pass across $V$.

We define a sequence of Riemannian structures $g_{p}=\left(1+\frac{\phi}{p}\right) g$. We will discuss two cases.

Case 1. For $p$ large enough, $y \in \mathfrak{A}_{x}^{g_{p}}$. By Lemma 10, there exists a $g$ segment $\sigma$ from $x$ to $y$ which does not pass across $V$. We have on one hand

$$
\mathfrak{D}_{x}^{g}=L^{g}(\sigma)=L^{g_{p}}(\sigma) \geq d^{g_{p}}(x, y)=\mathfrak{D}_{x}^{g_{p}} .
$$

On the other hand, as $g_{p} \geq g$, we have $\mathfrak{D}^{g} \leq \mathfrak{D}^{g_{p}}$. Hence $\mathfrak{D}_{x}^{g}=\mathfrak{D}_{x}^{g_{p}}$.

Let $\sigma^{p}$ be a $g_{p}$-segment from $x$ to $y$. Suppose that $\sigma^{p}$ passes across $V$, then

$$
\mathfrak{D}_{x}^{g_{p}}=L^{g_{p}}\left(\sigma^{p}\right)>L^{g}\left(\sigma^{p}\right) \geq d^{g}(x, y)=\mathfrak{D}_{x}^{g},
$$

which is in contradiction with (9). Hence $\sigma^{p}$ cannot pass across $V$,

$$
L^{g}\left(\sigma^{p}\right)=L^{g_{p}}\left(\sigma^{p}\right)=\mathfrak{D}_{x}^{g_{p}}=\mathfrak{D}_{x}^{g},
$$


and, by Hypothesis (ii), $\sigma_{p}$ is a $g$-segment such that $\left(\vec{\sigma}_{0}, \vec{\sigma}^{p}\right) \geq \varepsilon$. Now consider the process consisting on choosing a $g$-segment $\sigma_{0}$, and replacing $g$ by $g_{p}$. By repeating this process finitely many times, we would obtain a situation where no segment from $x$ to $y$ will exist. This is obviously impossible, so after finitely many steps, we obtain a Case 2 .

Case 2. We can select a subsequence of $\left(g_{p}\right)_{p}$ such that $y \notin \mathfrak{A}_{x}^{g_{p}}$. For each integer $p$, take a sequence $\left(g_{p, q}\right)_{q}$ of Riemannian structures of $\mathcal{S}_{x}$, converging to $g_{p}$ (this is possible because Lemmas 5 and 9 involve that $\mathcal{S}_{x}$ is dense in $G^{r}$ ). Let $y_{p, q}$ be the only $g_{p, q}$-antipode of $x$. By selecting suitable subsequences, we assume that each sequence $\left(y_{p, q}\right)_{q}$ is converging to a $g_{p}$-antipode, say $y_{p}$. We also can assume that $y_{p}$ tends to $y$. Let $\sigma^{p}$ be a $g_{p}$-segment from $x$ to $y_{p}$ which does not pass across $V$. By Lemma 6, each cluster point $\sigma$ of $\left(\sigma^{p}\right)_{p}$ is $g$-segment from $x$ to $y$, which does not pass across $V$. It follows that $\sigma^{p}$ and $\sigma$ are $g$-geodesics. By a variant of the first variation formula of arclength

$$
\begin{aligned}
\mathfrak{D}_{x}^{g_{p}}-\mathfrak{D}_{x}^{g} & =L^{g}\left(\sigma^{p}\right)-L^{g}(\sigma) \\
& =-g\left(\vec{\sigma}, \overrightarrow{y y_{p}}\right)+o\left(\overrightarrow{y y_{p}}\right),
\end{aligned}
$$

where $\overrightarrow{y y_{p}} \in T_{y} M$ is the tangent vector such that $y_{p}=\operatorname{Exp}_{y}\left(\overrightarrow{y y_{p}}\right)$. We denote by $\tau_{p}$ the $g$-norm of $\overrightarrow{y y_{p}}$, and put $u_{p}=\frac{\overrightarrow{y y_{p}}}{\tau_{p}}$. As $T_{y}^{1 g} M$ is compact, we can assume (otherwise select a subsequence) that $\left(u_{p}\right)$ is converging to a unit vector $u$. Equation (10) leads to

$$
\Phi \stackrel{\text { def }}{=} \lim _{p \rightarrow \infty} \frac{\mathfrak{D}_{x}^{g_{p}}-\mathfrak{D}_{x}^{g}}{\tau_{p}}=-g(\vec{\sigma}, u) .
$$

As $\Phi$ does not depend on $\left(\sigma_{p}\right)$, there are at most two possible values for $\vec{\sigma}$, say $\vec{\sigma}_{1}$ and $\vec{\sigma}_{2}$. By Hypothesis (i), we have

$$
\varlimsup_{p \rightarrow \infty} d^{\subset}\left(\vec{\Sigma}_{x y_{p}}^{g_{p}},\left\{\vec{\sigma}_{0}, \vec{\sigma}_{1}, \vec{\sigma}_{2}\right\}\right) \leq \frac{1}{4 n} .
$$

Hence, for $p$ large enough, we have

$$
d^{\subset}\left(\vec{\Sigma}_{x y_{p}}^{g_{p}},\left\{\vec{\sigma}_{0}, \vec{\sigma}_{1}, \vec{\sigma}_{2}\right\}\right) \leq \frac{1}{3 n} .
$$

In order to conclude, we claim that for $q$ large enough, we have

$$
\mu_{x}^{g_{p, q}} \leq d^{\subset}\left(\vec{\Sigma}_{x y_{p, q}}^{g_{p, q}},\left\{\vec{\sigma}_{0}, \vec{\sigma}_{1}, \vec{\sigma}_{2}\right\}\right) \leq \frac{1}{2 n} .
$$

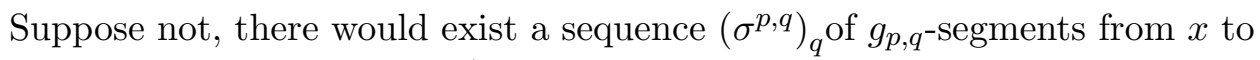
$y_{p, q}$ such that $\left(\overrightarrow{\sigma_{p, q}}, \vec{\sigma}_{i}\right)>\frac{1}{2 n}, 0 \leq i \leq 2$. A converging subsequence must tend to a $g_{p}$-segment $\sigma_{p}$ from $x$ to $y_{p}$ such that $\left(\overrightarrow{\sigma_{p}}, \vec{\sigma}_{i}\right) \geq \frac{1}{2 n}$, which is in contradiction with (12). 
Proof of Theorem 2. Take $(g, x) \in \mathcal{S}, x$ is joined to its only antipode by at most three segments if and only if $\vec{\Sigma}_{x a_{x}^{g}}^{g}$ has cardinality at most 3 , that is, by Lemma $2, \mu_{x}^{g}=0$. Let $T^{g}$ be the subset of $(M, g)$ of those points which are joined to their only antipodes by at least four segments. We shall prove that $G_{1}^{r} \stackrel{\text { def }}{=}\left\{g \in G^{r} \mid A_{2}^{g} \cup T^{g}\right.$ not meager $\}$ is meager. As

$$
G_{1}^{r} \subset\left\{g \in G^{r} \mid A_{2}^{g} \text { not meager }\right\} \cup\left\{g \in G^{r} \mid T^{g} \text { not meager }\right\},
$$

we only need to prove that $G_{2}^{r} \stackrel{\text { def }}{=}\left\{g \in G^{r} \mid T^{g}\right.$ not meager $\}$ is meager. As $T^{g}=\bigcup_{n \geq 1} \mathcal{V}^{g}(n)$ and $\mathcal{V}^{g}(n)$ is closed in $\mathcal{S}^{g}$, we obtain

$$
\begin{aligned}
G_{2}^{r} & \subset\left\{g \in G^{r} \mid \exists n \geq 1, \overline{\mathcal{V}^{g}(n)} \neq \emptyset\right\} \\
& =\bigcup_{n \geq 1}\left\{g \in G^{r} \mid \frac{\circ}{\mathcal{V}^{g}(n)} \neq \emptyset\right\} \\
& \subset \bigcup_{n \geq 1}\{g \in G^{r} \mid \overbrace{\mathcal{V}^{g}(n) \cup A_{2}^{g}} \neq \emptyset\} .
\end{aligned}
$$

Choosing a dense countable subset $S$ of $M$,

$$
\begin{aligned}
G_{2}^{r} & \subset \bigcup_{n \geq 1} \bigcup_{x \in S}\left\{g \in G^{r} \mid x \in \mathcal{V}^{g}(n) \cup A_{2}^{g}\right\} \\
& \subset \bigcup_{n \geq 1} \bigcup_{x \in S}\left\{g \in G^{r} \mid x \in \mathcal{V}^{g}(n)\right\} \cup \bigcup_{x \in S}\left\{g \in G^{r} \mid x \in A_{2}^{g}\right\} \\
& =\bigcup_{n \geq 1} \bigcup_{x \in S} \mathcal{V}_{x}(n) \cup \bigcup_{x \in S} G^{r} \backslash \mathcal{S}_{x} .
\end{aligned}
$$

By Lemmas 5 and $9, G^{r} \backslash \mathcal{S}_{x}$ is meager in $G^{r}$; by Lemmas 12 and 13 , $\bigcup \bigcup \mathcal{V}_{x}(n)$ is meager in $\mathcal{S}_{x}$ and consequently in $G^{r}$. $n \geq 1 x \in S$

\section{References}

[1] S. Bando and H. Urakawa, Generic properties of eigenvalues of the Laplacian for compact Riemannian manifolds, Tôhoku Math. J., 35 (1983), 155-172, MR 84h:58146.

[2] H.T. Croft, K.J. Falconer and R.K. Guy, Unsolved Problems in Geometry, SpringerVerlag, New-York, 1991, MR 92c:52001, Zbl 0748.52001.

[3] M. Gromov, J. Lafontaine and P. Pansu, Structure Métrique pour les Variétés Riemanniennes, Textes Mathématiques, CEDIC/Fenand Nathan, Paris, 1981, MR 85e:53051.

[4] J. Rouyer, En Géométrie Globale des Surfaces: La Notion d'Antipode, Thèse de Doctorat, 2001.

[5] _ On farthest points on Riemannian manifolds homeomorphic to the 2dimensional sphere, to appear in Rev. Roum. Math. Pures Appl. 
[6] C. Vîlcu, On two conjectures of Steinhauss, Geom. Dedicata, 79 (2000), 267-275, MR 2001f:52005, Zbl 0971.52003.

[7] T. Zamfirescu, Points joined by three shortest paths on convex surfaces, Proc. Amer. Math. Soc., 123(11) (1995), 3513-3518, MR 96a:52001, Zbl 0851.52003.

[8] _ Extreme points of the distance function on a convex surface, Trans. Amer. Math. Soc., 350(4) (1998), 1395-1406, MR 98i:52005, Zbl 0896.52006.

Received April 3, 2002 and revised November 4, 2002. Special thanks are due to the referee for pointing out some errors in a previous version of this paper.

Laboratoire de Mathématiques et Applications

Faculté des Sciences et Techniques - Université de Haute-Alsace

4, RUE DES FRÈRES LUMIÈRE

F-68 093 Mulhouse Cedex

FRANCE

E-mail address: J.Rouyer@uha.fr 